Apidologie, 1981, 12 (1), 31-36.

\title{
ESSAI PRÉLIMINAIRE DE TRAITEMENT \\ DE LA VARROASE (à VARROA JACOBSONI) \\ DE L'ABEILLE DOMESTIQUE PAR L'AMITRAZ
}

\author{
Vorläufige Behandlungsversuche der Varroatose \\ der Honigbiene (verursacht durch Varroa jacobsoni) mit Amitraz
}

par M. KILANI, J. BUSSIERAS (*), A. POPA et A. SAKLI (**)

\section{RÉSUMÉ}

L'amitraz utilisé en suspension aqueuse à la concentration de $0,001 \%$, permet de détruire la quasitotalité des Varroa présentes sur les abeilles adultes de ruchettes infestées, tout en ne conservant qu'une toxicité négligeable pour les abeilles elles-mêmes.

\section{SUMMARY}

A PRELIMINARY TRIAL ON THE TREATMENT OF VARROASIS (VARROA JACOBSONI) OF THE HONEY BEE BY AMITRAZ

A $0,001 \%$ aqueous suspension of amitraz, almost completely destroyed the Varroa present on the adult bees of infested hives, with only negligible toxicity of the bees themselves.

\section{INTRODUCTION}

La varroase, acariose épizootique récente de l'abeille domestique, s'étend rapidement à travers les continents et menace tous les élevages apicoles. Sa gravité, liée au

École nationale de Médecine vétérinaire, Sidi Thabet (Tunisie).

* École nationale vétérinaire, 94704 Maisons Alfort Cedex (France).

** Office tunisien de l'Élevage, La Soukra (Tunisie). 
développement du parasite en totalité à l'intérieur de la ruche, est augmentée par la difficulté du traitement.

Les échecs thérapeutiques sont en effet nombreux par suite de :

- la présence de stades de développement de Varroa jacobsoni dans le couvain operculé;

- la faible activité des médicaments actuellement disponibles quand ils ne sont pas toxiques pour les abeilles;

- l'apparition de phénomènes de résistance vis-à-vis de certains des produits utilisés.

Aussi une nouvelle substance du groupe des diamidides, l'amitraz*, qui paraît avoir une action acaricide majeure mais des propriétés insecticides très limitées, a-t-elle été expérimentée.

Le but de ce travail est d'évaluer dans un premier essai l'efficacité maximale de l'amitraz contre la varroase, compatible avec une toxicité minimale sur les abeilles.

\section{MATÉRIEL ET MÉTHODE}

Encore absente d'Europe occidentale, la varroase est actuellement très répandue en Afrique du Nord. Aussi l'expérimentation a-t-elle eu lieu dans les ruchers de l'Office Tunisien de l'Élevage à la Soukra (Tunisie), en mai 1980 .

L'expérimentation a consisté dans le traitement de différents lots d'abeilles enfermés dans des cagettes, avec l'amitraz à différentes concentrations.

a) Les lots d'abeilles: au nombre de 21. Chaque lot est composé d'environ 160 abeilles, prélevées le soir dans des ruchers expérimentaux.

b) Les cagettes sont en bois de dimensions $20 \mathrm{~cm} \times 15 \mathrm{~cm} \times 15 \mathrm{~cm}$ avec un trou pour nourrir les abeilles, deux parois vitrées coulissantes et un plancher grillagé. Elles sont disposées sur un papier enduit de vaseline.

c) L'amitraz : émulsion concentrée à $12,5 \%$ de principe actif (Taktic N.D.).

d) Les concentrations testées : de $0,1 \%$ à $0,001 \%$. Les lots ont été regroupés en trois séries successives : chaque série de 7 cagettes dont 1 témoin a permis de tester 3 concentrations différentes, soit 9 concentrations au total (voir tableau $n^{\circ} 1$ ).

e) Modes de traitement : un même volume de produit $(10 \mathrm{ml})$ de chaque concentration a été utilisé selon deux modalités de traitement différentes :

- par pulvérisation (P) à travers le plancher grillagé; cagette.

- et par évaporation (E) à partir d'une gaze imbibée, pliée dans un verre de montre et glissée dans la

f) Appréciation des résultats:

- le papier enduit de vaseline est enlevé et remplacé au bout de 24 heures et 48 heures. Le nombre de Varroa tombées est noté.

- Aux mêmes moments, les abeilles mortes sur le plancher de la cagette sont dénombrées et conservées.

\footnotetext{
* Fabriqué par The Boots Company, Nottingham (Angleterre).
} 
TABL. 1. - Modes d'application de l'amitraz et concentrations testées.

$\mathbf{P}=$ pulvérisation; $\mathbf{E}=$ évaporation.

TAB. 1. - Arten der Anwendung von Amitraz und geprüfte Konzentrationen.

$\mathbf{P}=$ Zerstäubung; $\mathbf{E}=$ Verdunstung.

\begin{tabular}{|c|c|c|}
\hline Série & $\begin{array}{l}\text { Mode d'application } \\
\text { Art der Anwendung }\end{array}$ & $\begin{array}{c}\text { Concentration amitraz \% } \\
\text { Konzentration von Amitraz (\%) }\end{array}$ \\
\hline 1 & $\begin{array}{c}\mathbf{P} \\
\mathbf{E} \\
\mathbf{P} \\
\mathbf{E} \\
\mathbf{P} \\
\mathbf{E} \\
\text { Témoin } \\
\text { Kontrolle }\end{array}$ & $\begin{array}{l}0,1 \\
0,1 \\
0,05 \\
0,05 \\
0,01 \\
0,01\end{array}$ \\
\hline 2 & $\begin{array}{c}\mathbf{P} \\
\mathbf{E} \\
\mathbf{P} \\
\mathbf{E} \\
\mathbf{P} \\
\mathbf{E} \\
\text { Témoin } \\
\text { Kontrolle }\end{array}$ & $\begin{array}{l}0,03 \\
0,03 \\
0,008 \\
0,008 \\
0,005 \\
0,005\end{array}$ \\
\hline 3 & $\begin{array}{c}\mathrm{P} \\
\mathbf{E} \\
\mathbf{P} \\
\mathrm{E} \\
\mathbf{P} \\
\mathrm{E} \\
\text { Témoin } \\
\text { Kontrolle }\end{array}$ & $\begin{array}{l}0,006 \\
0,006 \\
0,003 \\
0,003 \\
0,001 \\
0,001\end{array}$ \\
\hline
\end{tabular}

- Après 48 heures, toutes les abeilles sont comptées : après anesthésie au gaz carbonique $\left(\mathrm{CO}_{2}\right)$ elles sont disposées sur un tamis et plongées dans l'alcool à $95^{\circ} \mathrm{C}$.

- Sous le tamis, un tissu permet de recueillir les Varroa qui se détachent des abeilles dans le bain d'alcool : leur nombre total est noté.

\section{RÉSULTATS}

Voir tableau 2.

\section{DISCUSSION}

Le nombre de Varroa par 100 abeilles, en moyenne de 23, est relativement faible, probablement en raison de la saison du printemps au cours de laquelle le développement de l'acarien ne fait que débuter. 


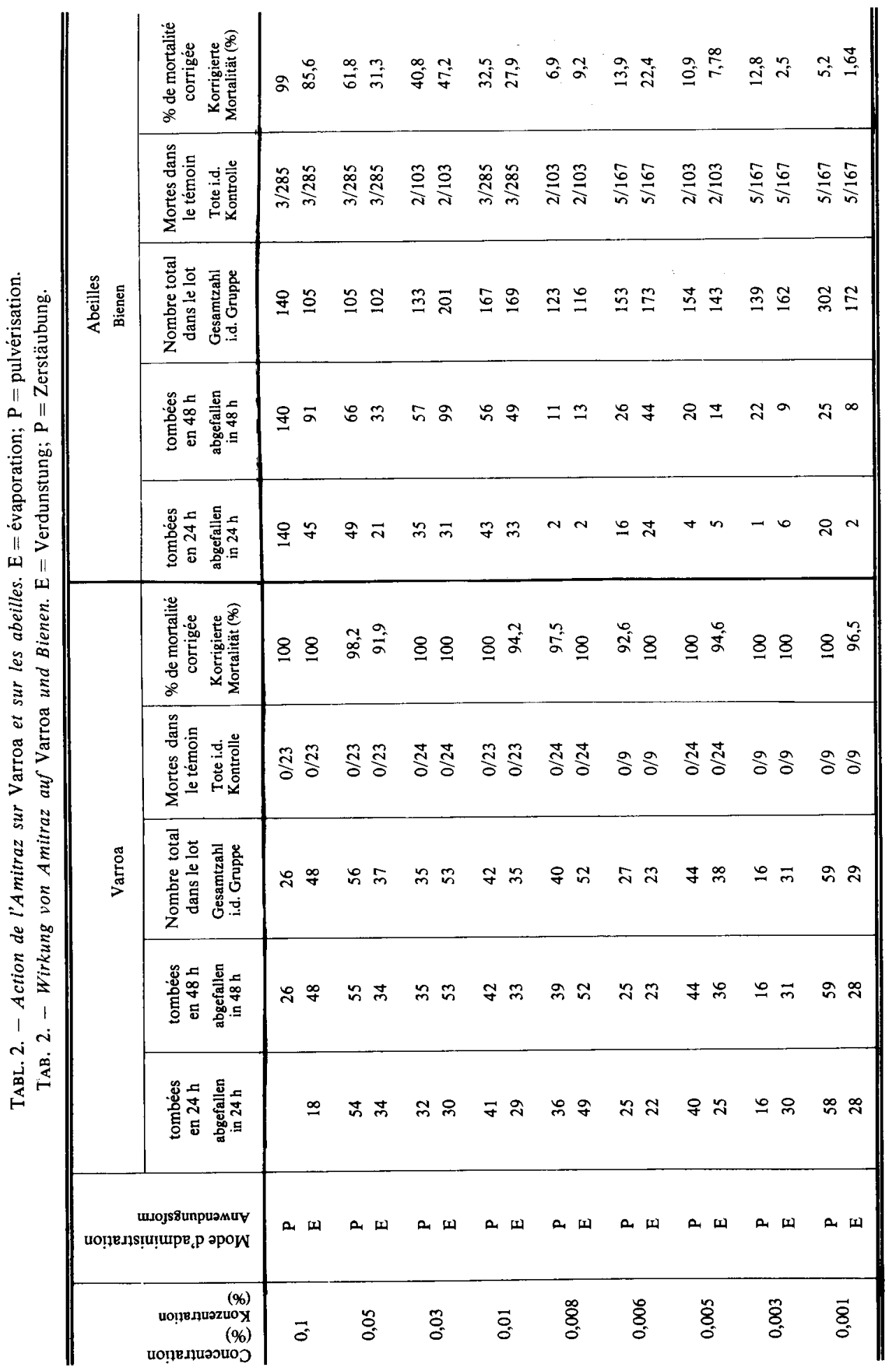


A la concentration minimale utilisée : $0,001 \%$ d'amitraz, $100 \%$ des Varroa sont tombées après pulvérisation, et $96,5 \%$ après évaporation du produit. A cette même concentration, $5,2 \%$ et $1,64 \%$ des abeilles ont été tuées respectivement.

L'amitraz allie donc une très bonne innocuité vis-à-vis des abeilles à une excellente efficacité contre les Varroa. Ce résultat est d'autant plus remarquable que nous avons utilisé une forme d'amitraz (le Taktic N.D.) destinée aux grands mammifères, et dont l'excipient n'est peut-être pas parfaitement adapté au traitement des abeilles.

Par ailleurs, la faible rémanence de l'amitraz fait espérer une toxicité négligeable du miel provenant de ruches traitées (mais seul un dosage des résidus permettra de conclure à ce sujet). Sans plus tarder, et en évitant les périodes de miellée, il sera intéressant de préciser les modalités pratiques de traitement dans un rucher d'exploitation, en tenant compte évidemment de la grosse difficulté liée à la présence des Varroa dans le couvain.

Enfin, l'excellente activité de l'amitraz contre la varroase, montrée dans ce premier essai, doit conduire à rechercher une efficacité éventuelle dans le traitement de l'acariose à Acarapis woodi.

Reçu pour publication en août 1980. Eingegangen im August 1980.

\section{ZUSAMMENFASSUNG}

Die Wirksamkeit von Amitraz, einem neueren Akarizid aus der Gruppe der Diamidide, auf die Varroatose der Honigbiene wurde untersucht. Amitraz ist als wenig bienengefährlich bekannt.

Bei den Versuchen, die in Tunesien durchgeführt wurden (einem Land, das von Varroa jacobsoni befallen ist) wurden Gruppen von etwa 160 adulten Bienen aus befallenen Völkern in vergitterten Käfigen gehalten. Die Käfige standen auf mit Vaseline beschichtetem Papier.

Jede Gruppe wurde mit $10 \mathrm{ml}$ einer wässrigen Suspension von Amitraz behandelt, entweder durch Zerstäuben durch das Gitter hindurch, oder durch Verdunstung. Die geprüften Konzentrationen bewegten sich zwischen 0,1 bis $0,001 \%$, bezogen auf den Wirkstoff.

Die Wirkung der Behandlung wurde nach folgenden Kriterien beurteilt :

- Zahl der Varroa-Milben, die innerhalb von $24 \mathrm{~h}$ und $48 \mathrm{~h}$ nach der Behandlung auf das Papier abgefallen waren.

- Zahl der durch die Behandlung getöteten Bienen.

- Zahl der Varroa-Milben, die $48 \mathrm{~h}$ nach der Behandlung lebend auf den Bienen gefünden wurden.

Die günstigsten Ergebnisse wurden bei der niedrigsten Dosierung $(0,001 \%)$ gefunden : getötet.

- Bei der Behandlung durch Verdunstung wurden 96,5\% der Varroamilben und 1,64\% der Bienen tet.

- Bei der Behandlung durch Zerstäuben wurden $100 \%$ der Varroamilben und 5,2\% der Bienen getö-

Diese Resultate erscheinen sehr ermutigend und sollten zur Prüfung der Wirksamkeit des Amitraz bei der Behandlung von Wirtschaftsvölkern eingesetzt werden, und zwar sowohl gegenüber Varroa jacobsoni wie gegenüber Acarapis woodi. 


\section{BIBLIOGR APHIE}

On trouvera une bibliographie détaillée sur la varroase dans :

APIMONDIA, 1977. - La varroase, maladie de l'abeille mellifère. - Bucarest, $100 \mathrm{p}$.

Chamoux H., 1977. - La varroase, une nouvelle et dangereuse parasitose des abeilles. Thèse Doct. Vèt., Alfort, $78 \mathrm{p}$.

El FourJı L., 1979. - La varroase, maladie de l'abeille mellifère. Thèse Doct. Vét., Sidi Thabet, 65 p. $\mathrm{N}^{\circ}$ spécial "Varroase " de Allgemeine deutsche Imkerzeitung (R.F.A.), 1980, nº 5. 